\title{
Erratum to: Brainstem raphe lesion in patients with major depressive disorder and in patients with suicidal ideation recorded on transcranial sonography
}

Mislav Budisic • Dalibor Karlovic - Zlatko Trkanjec • Arijana Lovrencic-Huzjan • Vlasta Vukovic • Jelena Bosnjak • Vida Demarin

Published online: 25 November 2009

(C) Springer-Verlag 2009

Erratum to: Eur Arch Psychiatry Clin Neurosci DOI 10.1007/s00406-009-0043-z

Author names were published in wrong order.

The online version of the original article can be found under doi: 10.1007/s00406-009-0043-z.

M. Budisic $(\square) \cdot$ Z. Trkanjec · A. Lovrencic-Huzjan .

V. Vukovic · J. Bosnjak · V. Demarin

Department of Neurology, University Hospital "Sestre

Milosrdnice", Vinogradska Cesta 29, Zagreb, Croatia

e-mail: mislavbudisic@yahoo.co.uk

D. Karlovic

Department of Psychiatry, University Hospital "Sestre

Milosrdnice”, Vinogradska Cesta 29, Zagreb, Croatia 